\title{
LA REVISTA DE LA FACULTAD DE DERECHO DE LA UNIVERSIDAD DEL CUSCO
}

Hace casi siete décadas, en 1948, comenzó a publicarse la REVISTA DE LA FACULTAD DE DERECHO Y CIENCIAS POLÍTICAS de la Universidad Nacional de San Antonio Abad del Cusco, formando parte de una tradición regional añeja de publicaciones sobre ciencias sociales y jurídicas, que proliferaron en Cusco desde fines del siglo XIX.

Fue una de las primeras, y quizás la única en su tiempo, que desde las universidades peruanas se ocuparon del campo jurídico de manera transformadora de las ciencias del Derecho desde una visión de vanguardia: con abundantes artículos que presentaron una visión crítica, renovada, multidisciplinar y de resaltante contenido sociojurídico. Tuvo una considerable difusión e impacto en el ámbito académico peruano y latinoamericano.

El camino que ha recorrido puede ser ordenado en cuatro etapas, en atención a los periodos históricos que la influyeron, al devenir ideológico que expresó y a las circunstancias sociales y políticas que la rodearon.

La primera etapa corresponde a la aparición de la primera Revista, en 1948. Entonces se percibían aún los ecos de las publicaciones que, desde 1890 hasta 1920, produjo el Colegio de Abogados del Cusco. En esta ciudad -de alrededor de dieciocho mil habitantes entonces- fueron editadas por dicho ente deontológico varios números de "El Debate Judicial" (1897) y la "Revista del Derecho" (1915) (RAMOS NÚÑEZ, 2000). Esta tendencia tuvo que haberse combinado con el impulso propio de la Reforma de 1909, y su resonante vanguardia intelectual, que agrupó su pensamiento en la Revista Universitaria empezada a publicarse en 1912. Los resultados positivos de la reforma universitaria cusqueña dieron fruto en la Facultad de Derecho de manera tardía, con la aparición de la primera Revista de la Facultad de Derecho. Fue durante el decanato de Cesar A. Muñiz que salió a la luz el primer número de 1948, bajo su dirección y su importante arrastre personal.

La segunda etapa comprende los números 2 (1964) al 50 (1965) que se publicaron cuando era decano Ferdinand Cuadros Villena, y al número 6o (1971) publicado bajo la dirección de Enrique Holgado Valer (PROGRAMA ACADEMICO DE 
DERECHO, 1971)(1). Esta etapa se relaciona a la llamada "edad de oro de la Facultad de Derecho" en la que logran conjugarse los impulsos progresistas de un grupo de profesores de izquierdas -encabezados por el mismo Cuadros Villena y otros docentes como Carlos L. Valer Portocarrero- con los fines de investigación propios de la universidad.

La idea que inspira los cinco números que componen esta etapa es la necesidad de crear una "conciencia jurídica nacional" para solucionar los grandes problemas del país. Para lograr este fin los intelectuales de la Facultad de Derecho plantean que debería superarse "el modelo de la universidad teologal que sirve únicamente a la profesionalización" (FACULTAD DE DERECHO, 1964:53-56). En la década de 1960, comenzaban a tomar nuevas energías las organizaciones y movimientos campesinos-indígenas que dieron lugar a la reforma agraria, por lo que los docentes de la Facultad de Derecho, advierten la necesidad de una renovación universitaria substancial más acorde a los tiempos de cambio de las estructuras sociales del Perú, y exponen que uno de los componentes indispensables de un nuevo tipo de Facultad, junto con la extensión universitaria, es la investigación de "la realidad socio-jurídica nacional y la formación de un derecho destinado a promover la transformación de la sociedad peruana y conseguir que se realicen los postulados de la democracia en los planos económico, político y cultural".(CUADROS VILLENA, 1964:58)

Los editores de la Revista conciben entonces que la Facultad de Derecho no puede "desentenderse de las múltiples exigencias de un mundo nuevo", sino que "debe contribuir a la creación y al imperio de un orden justo de vida, no pudiendo permanecer como simple espectadora de la vida nacional", sino que debe ser su protagonista. El propio Cesar A. Muñiz resalta que "toca en suma investigar y encontrar los ideales de la verdadera justicia social del pueblo peruano, contribuyendo también a la formación de un pensamiento filosófico jurídico propio de nuestra América Latina" (MUÑIZ, 1964:5-16).

El ímpetu transformador de la Revista de Derecho fue cercenado de manera abrupta por los hechos políticos que entonces envolvieron a la sociedad cusqueña. Durante la década del sesenta -como ya se advirtió- se producen importantes levantamientos campesinos indígenas, la ocupación - "toma de tierras" - de algunas haciendas, exigiendo la reforma agraria. El núcleo de docentes de la Facultad, editores

(1) A partir de este número las revistas de la Facultad ya son editadas como fruto del esfuerzo de un "Comité de Redacción"; al parecer dejan de ser parte del esfuerzo institucional de la Facultad para convertirse en fruto del emprendimiento de pequeños grupos de profesores sensibilizados con la producción intelectual como fin de la universidad. 
de la Revista, que impulsó y apoyó abiertamente este movimiento emancipador, fue perseguido por el régimen político, y varios de sus integrantes fueron confinados en las cárceles como el "Cepa" y el "Frontón" (TAMAYO HERRERA, 2013:49-50)

Luego de la represión contra los profesores de izquierdas, tuvieron que transcurrir seis años para que la Revista reapareciera en abril de 1971, con artículos que muestran las primeras influencias decididas de las teorías que conciben el estudio de la dogmática normativista del Derecho, la descripción de la ley, como contenido único y puro de la "ciencia jurídica". Aunque se aprecian todavía trabajos que buscan analizar la reforma de la educación decretada por el régimen de la Junta Militar de Gobierno y sus implicancias para la universidad peruana. Nuevamente los hechos políticos determinaron que la Revista dejara de ser publicada: en 1972, como respuesta a las potentes manifestaciones del movimiento estudiantil que reclamaban la recuperación de la democracia en el Perú, la UNSAAC fue intervenida por el gobierno militar mediante una "Comisión Reorganizadora".

La tercera etapa de la Revista de la Facultad de Derecho abarca los números 70 (1990) y 8 (1994) editados durante los decanatos de Florencio Díaz Bedregal y de José Béjar Quispe, respectivamente. Tuvieron que transcurrir 19 años de silencio en su publicación debido a que buena parte de la década del 70 la UNSAAC estuvo intervenida por el gobierno, regida por decretos del régimen militar, y fue recién en 1982 que se logra desprender de la tutela que violaba su autonomía, con la promulgación de la ley 23733 (Ley universitaria "Alayza-Sanchez").

La vuelta a la institucionalidad de la Facultad de Derecho tomó casi toda la década del 80. Una de las expresiones de esta vuelta a la normalidad es la Revista Número 7으 (1990), editada en homenaje al 199o aniversario de creación de la Facultad de Derecho (FACULTAD DE DERECHO, 1990)(2) con artículos más bien breves que abandonan -excepto los artículos sobre historia de la Facultad y el Paraninfo- los ánimos investigadores del binomio derecho y realidad social, que habían caracterizado a la segunda etapa de la Revista. En cambio, se percibe la influencia sobresaliente de la metodología del legalismo y el formalismo positivistas.

El número 80 (1997) sale a la luz después de transcurridos seis años de la publicación de su predecesora. No pudo cumplirse la intención institucional de que fuera anual, debido al recorte de presupuesto, "expresión de una práctica tradicional del gobierno peruano de no asignarle fondos suficientes a la Universidad pública" (BEJAR QUISPE, 1990).

(2) El "Consejo Editorial" de este número esta conformado por los docentes José Bejar Quispe y Luis A. Aragón Ibarra, además del Sr. Víctor Lovon Torres y el estudiante Elver Pizarro Pillco. 
Las revistas publicadas por los estudiantes. Es necesario mencionar un fenómeno académico muy importante: las revistas que salieron a la luz gracias al ímpetu de los estudiantes.

La primera de la que se tiene noticia es "Justicia" (1966), órgano del Centro Federado de Estudiantes de la Facultad de Derecho. Al parecer esta revista tuvo solo un número que puede ser clasificado dentro del grupo de publicaciones de la segunda etapa. Combina de muy buena manera trabajos de investigación de los estudiantes con artículos de contenido sociojurídico de profesores. Se puede destacar el artículo de Ferdinand Cuadros que hace una reflexión sobre "la ciencia jurídica al servicio del Perú como país en vía de desarrollo" en el que caracteriza el Derecho en el Perú como parcial, anacrónico, injusto; describe cómo la ley es construida a favor de las clases o grupos gobernantes y en contra del indígena; detalla el permanente retraso de la norma legal en relación al avance de la sociedad -"no suele el Derecho avanzar al compás el avance de la vida"-, y refiere como generalmente se promulga en contra de los intereses de la sociedad; razones por las que hace un llamado a la creación de una nueva "ciencia y conciencia para la libertad"(CUADROS VILLENA, 1966:3-28).

Un trabajo destacable, por su contenido de vanguardia, es "El problema sexual en las prisiones", de la profesora Bertha Degregori, en el que clama por que los gobernantes "den a los encarcelados un trato de seres humanos", que se solucionen, junto con la "ejecución de las penas, las prisiones, la arquitectura de éstas, la educación, el trabajo", los problemas sexuales. Desde un punto de vista freudiano propone estudiar la criminalidad femenina y el comportamiento sexual en las cárceles para proteger la estabilidad del recluso y de su familia y, en especial, proteger a la sociedad (DEGREGORI, 1966:28-54).

También contiene artículos de antropología jurídica. Uno de ellos es el de Jorge Flores Ochoa -entonces un novel profesor de la Facultad de Derecho- que diseña una guía para realizar estudios etnográficos del proceso judicial desde una visión que rompe con el positivismo normativista, con categorías propias de la antropología cultural (FLORES OCHOA, 1966:74-86). Otro artículo con esta temática es el de Rodolfo Sánchez: "Antropología, una perspectiva en la ciencia del Derecho" (SÁNCHEZ GARRAFA, 1966: 92-100). También es de resaltar el trabajo de Pedro Chalco Vizcarra que propone poner fin al dominio privado de las aguas que favorece su uso prioritario por los grandes terratenientes (CHALCO VIZCARRA, 1966:87-91).

Luego, ya durante la tercera etapa, se publican varias revistas dirigidas y gestionadas por los estudiantes de manera exclusiva. Una de las iniciales es "La Rotativa Jurídica" (1986) que alcanzó dos números con artículos de contenido políticojurídico enfocados en la coyuntura social (FACULTAD DE DERECHO, 1993). Se tocan, por ejemplo, temas como la militarización y la masacre de los penales en Lima (VALER DELGADO, 1993:18-21), el derecho socialista (MASIAS,1993:15-17), la deuda externa 
(LUNA FARFAN, 1993:25-30), y comienza a popularizarse el entendido de que la fundación de la Facultad de Derecho del Cusco data de 1791 (DIAZ BEDREGAL, 1993:714).

Otra de las publicaciones importantes de los estudiantes es la "Revista Jurídica Temas de Derecho" que produjo dos números, el primero editado en 1992 en homenaje al bicentenario de creación de la Facultad de Derecho y al tricentenario de fundación de la UNSAAC. Esta revista salió a la luz en medio del conflicto armado interno y de una de las más graves coyunturas económicas y sociales por las que atravesó el Perú, con la declarada finalidad de "formar investigadores jurídicos que planteen alternativas ante el sistema legal en crisis (...) y para tender vínculos de integración, comunicación e intercambio con quienes tienen que ver con el derecho y la sociedad" amplió su cobertura a las Facultades de Derecho del Sur del país(3) (TORRES ROSELLO, 1993).

En la primera década de este siglo aparece "Yachaq, Revista de Derecho" que alcanzó seis números (el último publicado en 2010) con artículos orientados a cuestiones de derecho privado y empresarial en su mayoría escritos por académicos de universidades limeñas, además de investigaciones sobre teoría y filosofía del derecho de autores extranjeros.

Desde mediados de los 80 , las revistas editadas por los estudiantes constituyen una tendencia significativa. La inquietud publicadora ha dado lugar a la organización de varios centros e institutos de investigación. Un hecho curioso, por ejemplo, es la "reaparición" de "La Rotativa Jurídica" en 2010, editada esta vez por el Instituto de Investigación de Derecho y Justicia (INSTITUTO DE INVESTIGACIÓN DE DERECHOYJUSTICIA, 2010)(4)

Hacia una cuarta etapa. Como se ha observado, existe una tradición substancial en la que puede inscribirse la publicación de la "Revista de la Facultad de Derecho y Ciencias Políticas". Fue comenzada bajo el impulso, entonces todavía dichoso, de la Reforma Universitaria de 1909. Sin embargo, cada vez que emprendía una nueva etapa editorial tuvo que dejar de publicarse durante varios años, por lo general debido a diversas circunstancias externas, económicas y políticas (como la persecución a los docentes progresistas, la falta de presupuesto y las "intervenciones" que sufrió la UNSAAC mediante "comisiones reorganizadoras" digitadas desde organismos del poder político centralista, y por personajes ajenos a la universidad).

(3) UNSAAC, Universidad Nacional del Altiplano (Puno), Universidad Andina del Cusco, Universidad Néstor Cáceres Velásquez (Juliaca) y Universidad de Apurimac (Abancay)

(4) Agradezco la colaboración del Carlos Rodriguez Casaverde, estudiante de la Facultad de Derecho, que me proporcionó un ejemplar de esta publicación. 
Debido a estos hechos sociales y políticos, y con el paso de los años, las investigaciones y publicaciones fueron escaseando, y peor aún abandonando la inspiración nacional que le imprimieron aquellos "soñadores provincianos" -como L. Velasco Aragón, J.G. Cosio, L.E. Valcarcel, L.F. Aguilar, U. García, E. Delgado Vivanco, R. Latorre, E. Kallata, C.F Cuadros- que se atrevieron a pensar con cabeza propia. En cambio devinieron a nutrirse -hasta convertirse en tradición- de concepciones importadas. En todo el Cusco, la producción intelectual fue decayendo desde 1950, junto con la economía regional, a medida que languidecía también la Universidad Nacional San Antonio Abad sometida a una larga crisis de la cual no acaba de salir por completo.

De manera lamentable, la Revista de la Facultad de Derecho y Ciencias Políticas dejó de ser publicada nuevamente durante un exorbitante tiempo, hace ya 19 años. Esas causas han infundido, en un sector de la Academia cusqueña, una especie de desánimo, de pereza intelectual y vacaciones de la creatividad que es necesario superar, más aún cuando la universidad es preciada ya no únicamente por la parca formación profesional que logra proveer, sino principalmente por su proyección social, su creación científica y sus labores de investigación, que son fines primordiales de su existencia.

La Revista de la Facultad de Derecho y Ciencias Políticas de la UNSAAC debe continuar con su noble tradición histórica y emprender en libertad, con una energía institucional renovada, emancipada ya de dictaduras y restricciones, una cuarta etapa de su edición.(5) La orientación hacia la calidad universitaria es una exigencia principal que nuestra sociedad hace a sus intelectuales, y parte de esa calidad son precisamente las publicaciones que debe producir la universidad. Por ello es necesario reemprender los esfuerzos de edición de la Revista, continuar con su antigua y venerable tradición para fundirla con las tendencias contemporáneas y los debates actuales del Derecho de nuestro siglo.

En el contexto de la mundialización, la Revista de la Facultad de Derecho y Ciencias Políticas, en su cuarta etapa, debe servir como una de las herramientas para superar la crisis universitaria, y principalmente como un espacio de reflexión, proyección y presentación a escala global de los avances en la investigación del campo jurídico, elaborados desde nuestra propia realidad, con participación de toda la comunidad, de manera técnica, sistemática y continuada.

(5) En esta cuarta etapa cuenta con el soporte importante, como equipo editorial, del Laboratorio de Derecho, Sociedad y Política, y con el apoyo de toda la comunidad de docentes, estudiantes y trabajadores administrativos de la Facultad de Derecho. 
Debe contribuir a desarrollar las corrientes jurídicas y políticas que han ayudado a su existencia, además de abrirse a las nuevas tendencias de pensamiento. Así, la Revista deberá contener enfoques desde todas las maneras de estudiar el Derecho, es decir desde las ópticas de todas las ciencias jurídicas.(6) Su finalidad será servir a la construcción de un Estado constitucional y democrático, a la profundización de la autonomía personal, a la ampliación de la participación ciudadana y, sobretodo, a la libertad final de nuestra América, al logro de la justicia y la emancipación de nuestros pueblos.

Pável H. Valer Bellota Director de la Revista de la Facultad de Derecho y Ciencias Políticas

(6) En esto concordamos con que "el Derecho es estudiado en lo relativo a la estructuración lógica de sus normas, teniendo aplicación en ello las diversas corrientes racionalistas jurídicas; o bien puede ser estudiado su rol social, que es la perspectiva de las corrientes sociológicas; o también puede estudiarse su lenguaje o sus símbolos, desde el punto de vista de la semiótica, etc." [DE LA TORRE RANGEL, Jesús Antonio; El derecho como arma de liberación en América Latina: Sociología jurídica y uso alternativo del derecho; Facultad de Derecho de la Universidad Autónoma de San Luis Potosí; México, 2006. Pág. 37.] 


\section{Bibliografía}

BEJAR QUISPE, José (1990); "Prologo"; en: FACULTAD DE DERECHO; Revista de la Facultad de Derecho y Ciencias Políticas, Nro. 8, Universidad de San Antonio Abad del Cusco.

CHALCO VIZCARRA, Pedro (1966); "La subsistencia del dominio privado sobre las aguas en el Perú"; Justicia, Órgano del Centro Federado de Derecho; UNSAAC, Cusco, 1966, págs. 87-91.

CUADROS VILLENA, Carlos Ferdinand (1966,); "Ciencia jurídica al servicio del Perú como país en vía de desarrollo"; Justicia, Órgano del Centro Federado de Derecho; UNSAAC, Cusco, págs. 328.

CUADROS VILLENA, F. (1964); "La investigación de la realidad socio-jurídica, fin primordial de las Facultades de Derecho", proyecto de resolución en la I Convención de las Facultades de Derecho del Perú; en Revista de la Facultad de Derecho, Nro. 2, Cusco. Pág. 58.

DE LA TORRE RANGEL, Jesús Antonio (2006); El derecho como arma de liberación en América Latina: Sociología jurídica y uso alternativo del derecho; Facultad de Derecho de la Universidad Autónoma de San Luis Potosí; México, Pág. 37.

DEGREGORI de NIETO, Bertha (1966). "El Problema sexual en las prisiones" Justicia, Órgano del Centro Federado de Derecho; UNSAAC, Cusco, págs. 28-54.

DIAZ BEDREGAL, Florencio (1993). "Notas sobre la enseñanza del derecho en la Universidad Nacional de San Antonio Abad del Cusco"; en: FACULTAD DE DERECHO; La Rotativa Jurídica, Revista semestral, Añol, Nro. 2, Hernan Mellado Villafuerte (Dir.); Cusco 20/10/93. Págs. 7-14.

FACULTAD DE DERECHO (1964). "Objeto y fines de la Facultad de Derecho", proyecto de resolución en la I Convención de las Facultades de Derecho del Perú; en Revista de la Facultad de Derecho, Nro. 2, Cusco. Págs. 53-56

FACULTAD DE DERECHO (1990). Revista de la Facultad de Derecho y Ciencias Políticas, Nro. 7, Universidad de San Antonio Abad del Cusco,. El "Consejo Editorial" de este número está conformado por los docentes José Bejar Quispe y Luis A. Aragón Ibarra, además del Sr. Víctor Lovon Torres y el estudiante Elver Pizarro Pillco.

FACULTAD DE DERECHO (1993); La Rotativa Jurídica, Revista semestral, Año I, Nro. 2, Hernan Mellado Villafuerte (Dir.); Cusco 20/10/93.

FLORES OCHOA, Jorge (1966). "Bases para investigar procesos judiciales"; Justicia, Órgano del Centro Federado de Derecho; UNSAAC, Cusco, págs. 74-86. 
INSTITUTO DE INVESTIGACIÓN DE DERECHO Y JUSTICIA (2010); Revista la Rotativa Jurídica; Nro. 1, Ronald Hancco Lloclle (Dir); Cusco.

LUNA FARFAN, Faustino (1993); "La deuda externa y el derecho financiero y tributario", en: FACULTAD DE DERECHO; La Rotativa Jurídica, Revista semestral, Año I, Nro. 2, Hernan Mellado Villafuerte (Dir.); Cusco 20/10/93. Págs. 25-30.

MASIAS Z., Demetrio (1993); "El sistema jurídico socialista"; en: FACULTAD DE DERECHO; La Rotativa Jurídica, Revista semestral, Año I, Nro. 2, Hernán Mellado Villafuerte (Dir.); Cusco 20/10/93. Págs. 15-17.

MUÑIZ R., César A. (1964); "Fines de las Facultades de Derecho", Revista de la Facultad de Derecho, Nro. 2, Cusco 1964. Págs. 5-16.

PROGRAMA ACADEMICO DE DERECHO (1971). Revista de la Facultad de Derecho, Nro. 6, Cusco.

RAMOS NÚÑEZ, Carlos A. (2000). Historia Del Derecho Civil Peruano: Siglos XIX y XX; Fondo Editorial PUCP, Lima.

SÁNCHEZ GARRAFA, Rodolfo (1966); "Antropología, una perspectiva en la ciencia del Derecho"; Justicia, Órgano del Centro Federado de Derecho; UNSAAC, Cusco, págs. 92-100.

TAMAYO HERRERA, José (2013); "Historia integral de la Universidad Nacional de San Antonio Abad del Cuzco: nueva visión sintética desde la perspectiva teórica del siglo XXI"; en El Antoniano, Nro. 122, UNSAAC, Marzo de. Págs. 23-57. [p.49-50]

TORRES ROSELLO, Juan Carlos (1992). "Nota Editorial"; en Revista Jurídica Temas de Derecho; Consejo de Investigación de la UNSAAC, Facultad de Derecho y Ciencias Políticas; Cusco.

VALER DELGADO, Vladimiro (1993). "Las masacres en los penales de Lima: genocidio exponente de un proceso de autoritarismo y militarización"; en: FACULTAD DE DERECHO; La Rotativa Jurídica, Revista semestral, Año I, Nro. 2, Hernan Mellado Villafuerte (Dir.); Cusco 20/10/93. Págs. 18-21. 